\title{
Novel Technology Based on the Spectral Kurtosis and Wavelet Transform for Rolling Bearing Diagnosis
}

\author{
Len Gelman ${ }^{1}$, Tejas H. Patel ${ }^{2}$, Gabrijel Persin ${ }^{3}$, and Brian Murray ${ }^{4}$ Allan Thomson ${ }^{5}$ \\ ${ }^{1,2,3}$ School of Engineering, Cranfield University, Cranfield, Bedfordshire, MK430AL UK. \\ 1.gelman@cranfield.ac.uk, t.patel@cranfield.ac.uk, g.persin@cranfield.ac.uk, \\ ${ }^{4,5} S K F, U K$ \\ brian.murray@cranfield.ac.uk, allan.thomson@cranfield.ac.uk
}

\begin{abstract}
A novel diagnosis technology combining the benefits of spectral kurtosis and wavelet transform is proposed and validated for early defect diagnosis of rolling element bearings. A systematic procedure for feature calculation is proposed and rules for selection of technology parameters are explained. Experimental validation of the proposed method carried out for early detection of the inner race defect. A comparison between frequency band selection through wavelets and spectral kurtosis is also presented. It has been observed that the frequency band selected using spectral kurtosis provide better separation between healthy and defective bearings compared to the frequency band selection using wavelet. In terms of Fisher criterion the use of spectral kurtosis has a gain of 2.75 times compared to the wavelet.
\end{abstract}

\section{INTRODUCTION}

The use of wavelets (Nikolaou and Antoniadis, 2002; Qiu, Lee, Lin, and Yu, 2006; Klepka, 2011) and spectral kurtosis (Sawalhi and Randall, 2004; Antoni, 2006; Wang and Liang, 2011) is widely reported for condition monitoring of the rolling element bearing defect. These tools are often used to detect demodulation frequency bands for final diagnosis from the envelope spectrum. Nikolaou and Antoniadis (2002) presented the wavelet based demodulation scheme with automatic selection of the wavelet parameters based on Shannon entropy and magnification factor criterion. Qiu et al (2006) presented wavelet filter based signature detection for rolling element bearings. They designed a daughter Morlet wavelet with specific shape factor at certain scale/frequency so to improve detection of impulses due to defect from noisy

\footnotetext{
L. Gelman et al. This is an open-access article distributed under the terms of the Creative Commons Attribution 3.0 United States License, which permits unrestricted use, distribution, and reproduction in any medium, provided the original author and source are credited.
}

signal. Klepka (2011) also used wavelet for demodulation for bearing fault detection. The diagnosis was from the discrete Fourier transform of the wavelet coefficients. Sawalhi and Randall (2004) illustrated the use of the spectral kurtosis (SK) for bearing diagnostics. They have discussed a number of extensions, improvements and choice of optimum analysis parameters. Antoni (2006) discussed use of SK for vibratory surveillance and diagnostics of different rotating machines. They proposed the use of kurtogram to find parameters for optimal band pass filters. Wang and Liang (2011) proposed SK technique that adaptively determines the optimal bandwidth and center frequency through window construction by superposition.

Use of wavelets over SK has an advantage through variable time-frequency resolution at different frequencies, which enables detection of the closely spaced narrow band demodulation frequency bands. However, this requires proper selection of wavelet parameters through iterations which adds up the computation time. The SK on other hand is adaptive, computationally less expensive and fast, which makes SK ideal candidate to be incorporated into portable instrument for on-field applications in semi-automatic mode.

In the study here we present a novel technology that combined the SK and wavelet. The SK is used for detection of the demodulation frequency bands and wavelet is used for diagnosis through a diagnostic feature in the form of normalized integrated wavelet modulus. The processing is done of successive realizations obtained from the whole signal with a specific time duration corresponding to slowest possible modulating frequency. The proposed method thus takes care of the stochastic nature of bearing defect signals and slippage of the rolling element between races. The proposed novel method is compared with the wavelet technology. 


\section{EXPERIMENTAL SETUP}

The bearing test rig has a coupled VSD (variable speed drive) motor driving a shaft supported on three identical bearings (FK UCP203). Figure 1 shows the test rig under consideration. The VSD drive provides $20-60 \mathrm{~Hz}$ supply frequency. The test rig has provision for inducing load at the non-drive end (NDE). Tests were conducted at one speed and two levels of load, resulting into total two test conditions, namely, (1) full speed and full load (FSFL), and (2) full speed and half load (FSHL). The full speed and full load condition corresponds to $60 \mathrm{~Hz}$ supply frequency and $196 N$ resultant radial load.

The test bearing was placed on the non-drive end (NDE). The drive end (DE) and middle bearings were never changed during experiments. Table-1 lists the bearings geometric dimensions and the characteristic frequencies related to defect of inner race $(B P F I)$, outer race $(B P F O)$, cage $(F T F)$ and ball/roller $(B S F)$.

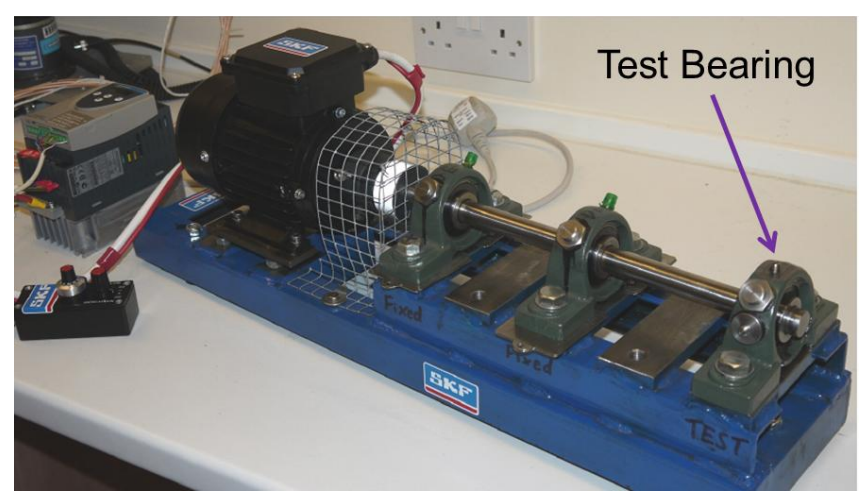

Figure 1. The bearing test-rig

\begin{tabular}{cc}
\hline Number of balls & 8 \\
\hline Bore diameter & $17 \mathrm{~mm}$ \\
\hline Outside diameter & $40 \mathrm{~mm}$ \\
\hline Ball diameter & $6.75 \mathrm{~mm}$ \\
\hline $\begin{array}{c}\text { Ball pass frequency, } \\
\text { inner race, } B P F I\end{array}$ & $4.95 * f_{r}$ \\
\hline $\begin{array}{c}\text { Ball pass frequency, } \\
\text { outer race, } B P F O\end{array}$ & $3.05 * f_{r}$ \\
\hline Ball spin frequency, $B S F$ & $1.99 * f_{r}$ \\
\hline $\begin{array}{c}\text { Fundamental train frequency, } \\
\text { FTF }\end{array}$ & $0.382 * f_{r}$ \\
\hline \multicolumn{2}{c}{$f_{r}$ is the shaft rotation frequency }
\end{tabular}

Table 1. Test bearing specifications and frequencies
Two sets of experiments were conducted, first with healthy bearing and second with the defective bearing. Figure 2 show pictures of a bearing with single inner race defect. The inner race defect is created in the form circumferential groove of $0.8 \mathrm{~mm}$ length and $0.2 \mathrm{~mm}$ deep. The relative damage size of inner race defect is $1.2 \%$ of circumference; so, it is an early stage of fatigue damage development. Vibration signals were captured from a unidirectional accelerometer placed along vertical direction on the test bearing housing. Active antialiasing filters were used before capturing digitized data. A speed reference signal (1 pulse per shaft revolution) was also captured synchronously. The data acquisition was done at $40 \mathrm{kHz}$ in order to provide necessary bandwidth for channel recording.

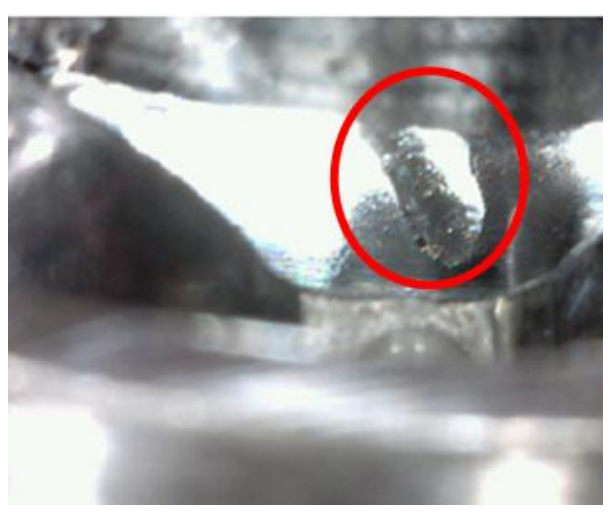

Figure 2. Inner race defect

\section{The Wavelet Diagnostic Feature}

The continuous wavelet transform (CWT) of signal $x\left(t^{\prime}\right)$ can be presented in the form (Gryllias, Gelman, Shaw, and Vaidhianathasamy, 2010);

$W(a, t)=\frac{1}{\sqrt{a}} \int_{-\infty}^{\infty} x\left(t^{\prime}\right) \psi^{*}\left(\frac{t^{\prime}-t}{a}\right) d t^{\prime}$

where, $\psi$ is the mother wavelet function, $a$ and $t$ are scale and time shift variables, ${ }^{*}$ denotes the complex conjugate.

The complex Morlet wavelet function used in this study is

$\psi\left(t^{\prime}\right)=\frac{1}{\sqrt{\pi f_{b}}}\left(e^{-j 2 \pi f_{c} t^{\prime}}-e^{-f_{b}\left(\pi f_{c}\right)^{2}}\right) e^{-t^{2} / f_{b}}$

where, $f_{c}$ is the central frequency of the mother wavelet function, $f_{b}$ is the bandwidth parameter, and $j$ is the imaginary quantity.

A normalized diagnostic feature used here is,

$I_{W}(t)=\frac{1}{B} \int_{f_{1}}^{f_{2}}|W(f, t)|^{2} d f$

where, $|W(f, t)|$ is the modulus of wavelet transform. $B=$ $\left[f_{1}, f_{2}\right]$ is the wavelet modulus integration frequency band. The band $B$ is the frequency band excited by the bearing defects. They are basically demodulation bands obtained 
using spectral kurtosis based optimal denoising filter. Instead of computing envelope of the signal, an instantaneous normalized wavelet modulus is calculated for the fraction of the signal. The diagnostic feature value will be high for the defective bearings compared to healthy bearings.

\section{METHOdOLOGY FOR FEATURE ESTIMATION}

In the following we describe the stepwise procedure for estimation of the wavelet diagnostic feature. The flowchart (Figure 3) illustrates steps of the proposed methodology.

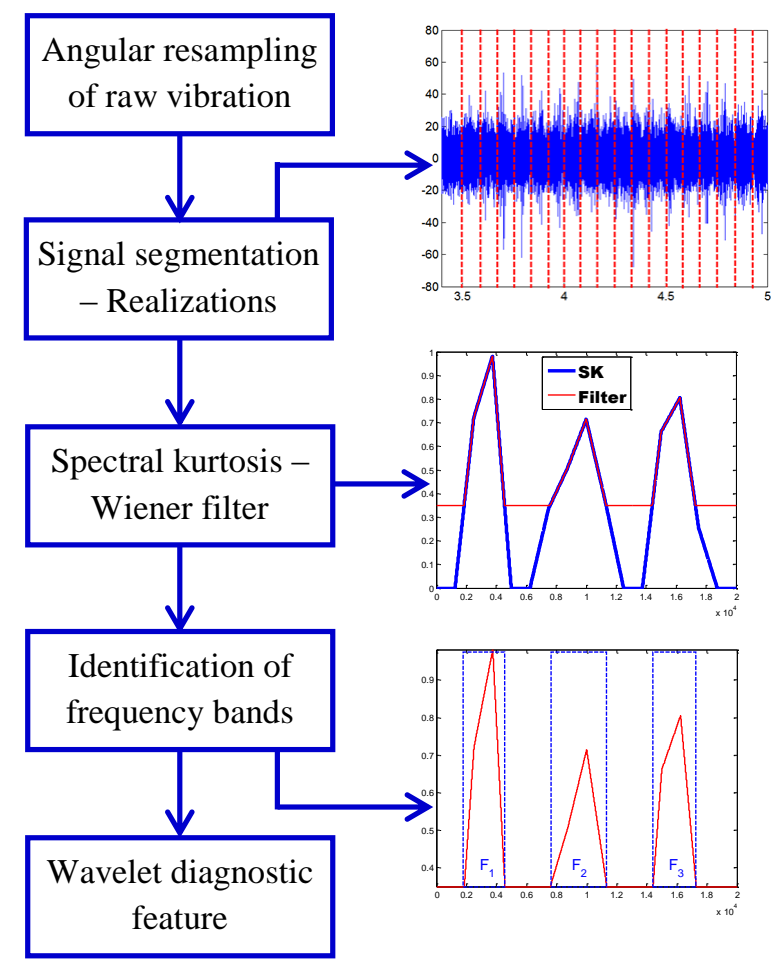

Figure 3. Flowchart of the proposed technology

1. Re-sample the vibration signal in the angular domain

The raw vibration signal was first resampled in the angular domain to compensate the slight fluctuation in the rotational speed and to ensure integer nos. of samples per rotation period. It is known [1-3] that the vibration signal for inner race (IR) defect depicts $B P F I$ and its harmonics, with defect frequencies being modulated by shaft frequency $\left(f_{r}\right)$ under stationary loading. In order to cover shaft rotation period and impacts from all 8 balls, the resampling was done with respect to $\left(f_{r}-F T F\right)$.

2. Signal segmentation - slicing into realizations
The angularly resampled signal was then sliced into realizations, with each realization equal to $1 /\left(f_{r}-F T F\right)$ seconds. Each realization was bigger than modulation period $\left(1 / f_{r}\right)$ and cover impacts from all 8 balls.

3. Spectral kurtosis and impact related frequency band detection

SK extends the statistical measure of the kurtosis to the function of frequency and indicates how the impulsivity in the signal is distributed in the frequency domain. SK can be used to design detection filters since it takes large values at frequencies where impulsivity is dominant and low values where there is Gaussian noise only. The resonant frequency bands were identified using the Spectral Kurtosis (SK) based optimal denoising (Wiener) filter for each realization. The procedure for obtaining SK based denoising filter is widely reported [4,5], therefore, related discussion omitted here. Since the impact duration is much shorter than the time between impacts, a window length of $1 / 10$ times the duration between impacts is taken for spectral kurtosis calculation, which corresponds to frequency resolution of 10 times of bearing characteristic defect frequency. Frequency bands $\left(\mathrm{F}_{\mathrm{i}}\right)$ were identified by comparing the SK to the significance threshold of $1 \%$, used to obtain the Wiener filter from the spectral kurtosis. The filter is used to determine the frequency bands, corresponding to significantly high values of SK and frequency bands excited by a defect. Using this method often more than one frequency bands were identified for defect signal (e.g. $F_{1}, F_{2}$, and $F_{3}$ in Figure 3 ). On the other hand, for no-defect case, the SK values would be below the threshold and therefore no frequency bands will be selected for majority of the realizations.

4. Calculation of wavelet feature (Eq. 3).

The estimation of the wavelet diagnostic feature (Eq. (3)) requires a choice of center frequency $\left(f_{c}\right)$ and bandwidth parameter $\left(f_{b}\right)$. For impact detection through vibration signal, $f_{c}=5$ to $6 \mathrm{rad} / \mathrm{sec}$ has been considered ideal; consequently $f_{c}=5 \mathrm{rad} / \mathrm{sec}$ chosen for this study. The impact duration is usually shorter than the time period between the impacts. Although it is difficult to estimate the exact impact duration, duration of $t_{i} / 8 \quad\left(t_{i}\right.$ is the time between impacts) can be considered minimum. With $f_{c}=5 \mathrm{rad} / \mathrm{sec}$, the choice of proper $f_{b}$ will balance between time and frequency resolution of the wavelet transform. The parameter $f_{b}$ should be chosen such that the wavelet transform provide minimum set time resolution, i.e. $t_{i} / 8$, at the lowest frequency band excited due to bearing defect impact. From study of few frequency bands from spectral kurtosis, it was found that the frequency of $1850 \mathrm{~Hz}$ is the lowest frequency; accordingly $f_{b}$ is set equal to $4 \sec ^{2}$. 
The wavelet transform is obtained for each realization for no-defect and defect cases. The instantaneous wavelet diagnostic feature was calculated for each sample of the realization for each frequency band identified in step \#2 above. The final diagnostic feature, i.e. normalized instantaneous wavelet modulus, is the cumulative sum of the wavelet features obtained in different frequency bands. In absence of any frequency band for a realization, the feature is assigned a zero value.

The study presented in this paper is novel from two aspects. One, the spectral kurtosis and wavelet technologies are combined. The second, the proposed technology requires a small signal length, i.e. only few realizations for bearing diagnosis.

This methodology mirrors the similar methodology of wavelet technology proposed and validated for bearing diagnosis by Gelman et al (2013). The only difference is in selection of impact related frequency bands. The method presented by Gelman et al (2013) needs historical data for frequency band selection through comparison of wavelet scalograms of defective and healthy bearings (Figure 4); whereas the SK based method presented here do not require any historical data. The selected frequency bands from wavelets are shown in blue boxes in Figure 4.

For comparison of SK based frequency band selection with the wavelet based frequency band selection, the Fisher criterion (Eq. (4)) of the diagnostic feature will be obtained and compared. The Fisher criterion is a statistical measure indicating separation between damaged and undamaged conditions.

$F C(\theta)=\frac{\mu_{D}(\theta)-\mu_{N D}(\theta)}{\sigma_{D}^{2}(\theta)+\sigma_{N D}^{2}(\theta)}$

where $\mu$ and $\sigma$ are respectively the mean and standard deviation of the integrated wavelet features estimated for each angle $\theta$ over all realizations, suffixes $D$ and $N D$ refer to the damaged and undamaged conditions.

\section{APPLiCATION TO DETECTION OF INNER RACE DEFECT}

The captured vibration signals for no-defect and inner race (IR) defect cases were first re-sampled in angular domain using a speed reference signal. To identify frequency bands excited by the defect, spectral kurtosis was calculated for each realization and optimal denoising filter was obtained using parameters stated in Section 4. Figure 5 shows the resonant frequency bands for first 500 realizations of the signal at full speed and full load condition (FSFL). Three frequency bands, i.e. around $5 \mathrm{kHz}, 11 \mathrm{kHz}$ and $16 \mathrm{kHz}$ were often detected for defective bearing. Similar frequency bands were identified for the other three test conditions.
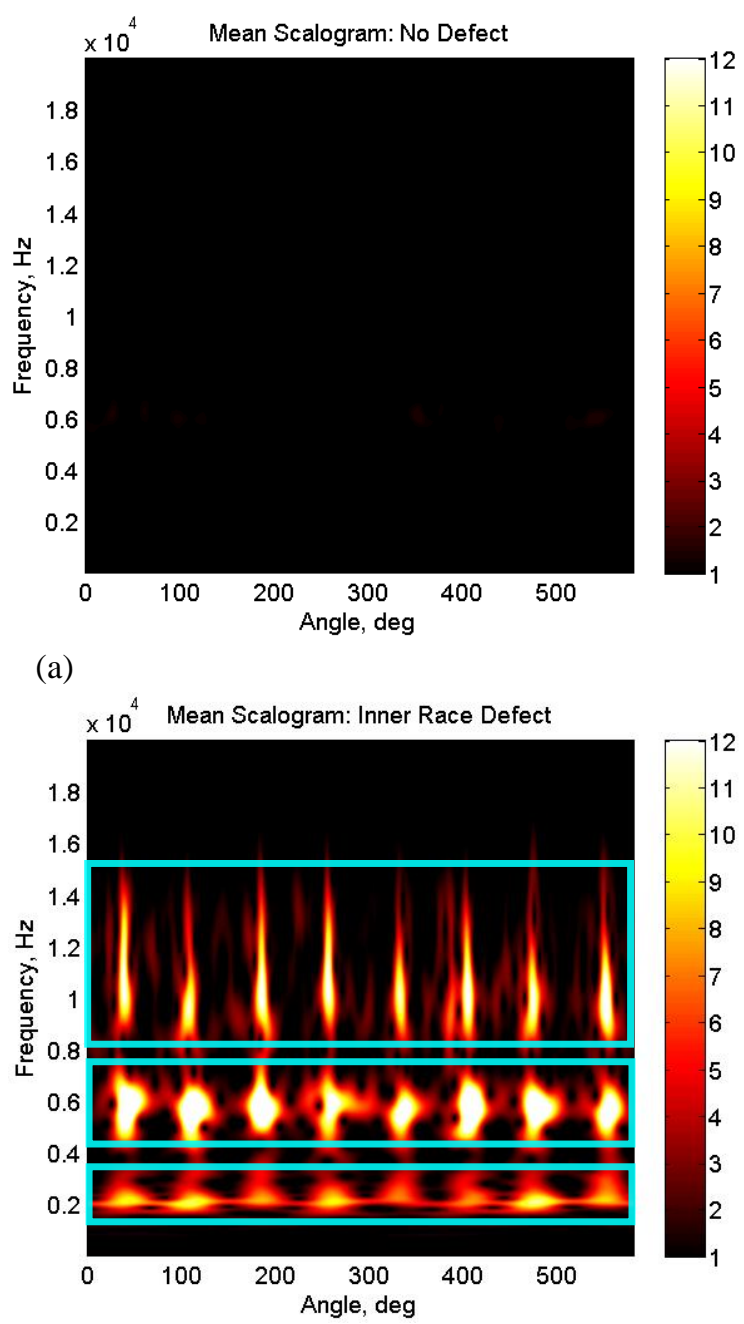

(b)

Figure 4

These frequency bands were used as integration bands $B$ for instantaneous integrated wavelet moduli (Eq. (3)). After computing wavelet feature for each of these bands, they were added to get a single cumulative diagnostic feature.

Figure 6 shows the diagnostic feature (i.e. cumulative integrated wavelet modulus) for 500 realizations of nodefect (Figure 6(a)) and inner race defect (Figure 6(b)) at FSFL condition. In the feature map (Figure 6(b)), lines with high value of features are visible. It is important to realize that the diagnostic feature has the high value for the impact and low value between two impacts. At particular period, 8 impacts from 8 balls are visible. These lines are inclined and not perfectly straight. This is due to slip of balls between bearing raceways. The Figure 6 also shows the benefit of realization wise calculation of wavelet transform and the diagnostic feature, the feature would have low value if averaged over realizations. 
Although the impacts were clearer, to improve the visibility of impacts further alignment of the feature was done using correlation property. To start with, realization \#1 of the feature map (Figure 6(b)) was taken as the reference to align realization \#2 so to have maximum correlation between these two realizations. Next, the mean of realization \#1 and aligned realization \#2 was used to align the realization \#3. This process was repeated till the last realization aligned. It is important to note that the alignment using correlation property essentially make the inclined lines of impacts in Figure 6(b) vertically straight (Figure 7). For no-defect case the feature value was either zero or very low, the alignment did not reveal any noticeable difference hence not shown.

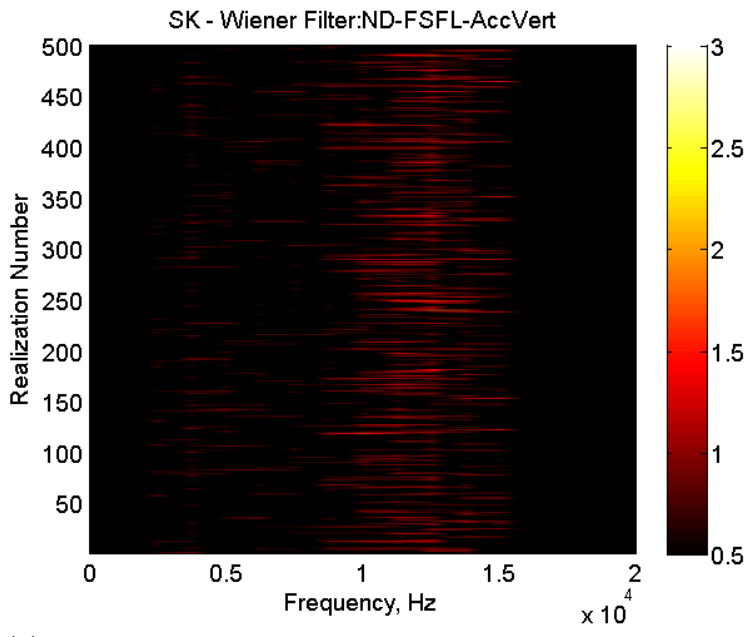

(a)

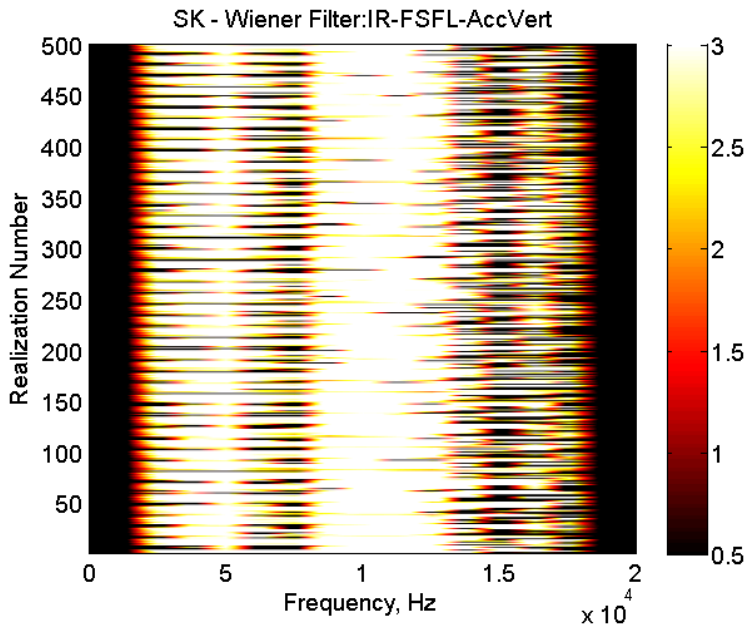

(b)

Figure 5

Figure 8 compares the wavelet diagnostic features for the defective bearing from the wavelet based frequency band selection proposed by Gelman et al (2013), and the SK based frequency band selection proposed in this paper. The wavelet based frequency band selection (Figure 8(a)) has high feature values, however with more noise, compared to the SK based frequency selection (Figure 8(b)). Figure 9 shows the comparison of both the frequency band selection method in terms of Fisher criterion (Eq. (4)) of the diagnostic feature. Eight peaks for eight impacts are clearly visible in Figure 9(a). In terms of Fourier transform amplitude (Figure 9(b)) at the defect frequency, i.e. amplitude at frequency order 8 , the SK based frequency band selection has the gain of 2.65 times compared to the wavelet based frequency band selection.

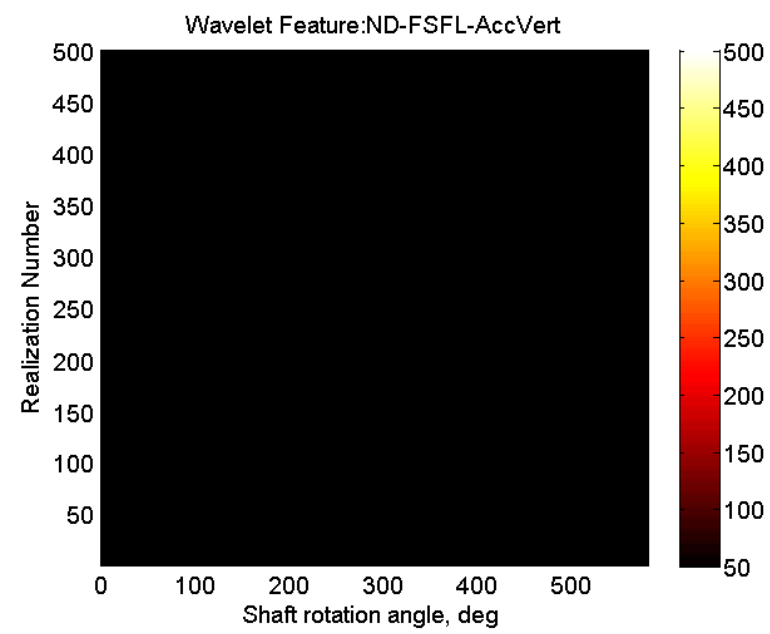

(a)

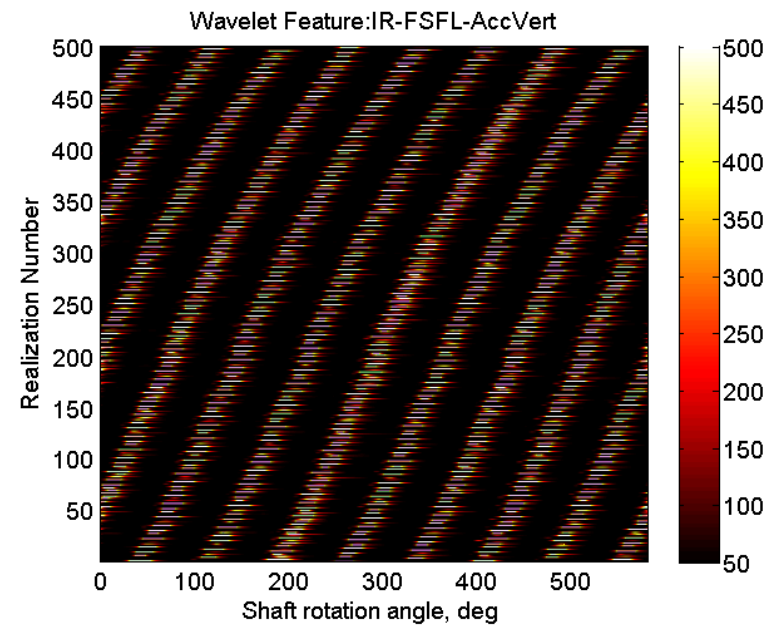

Figure 6 


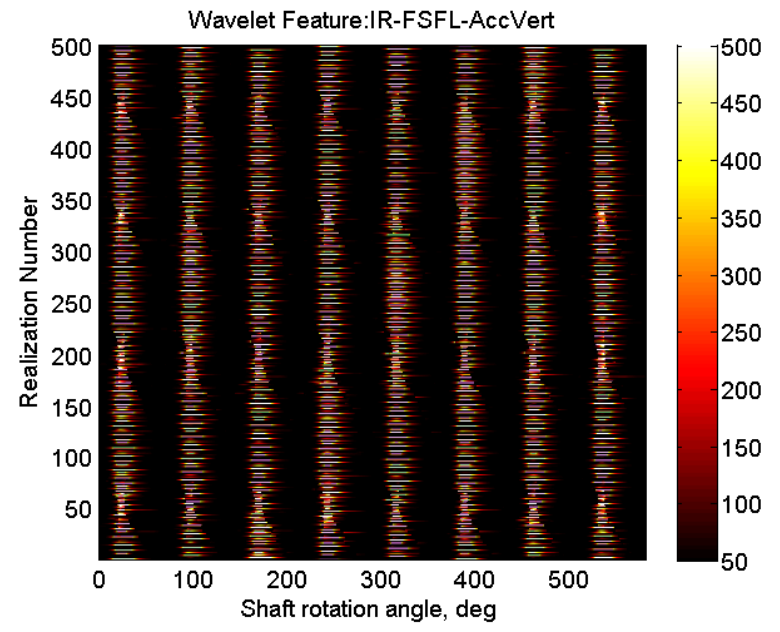

Figure 7

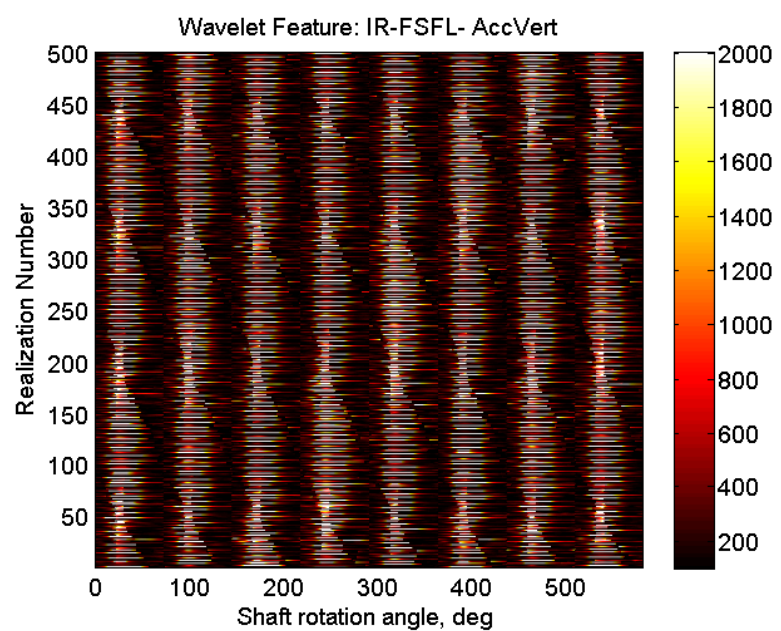

(a)

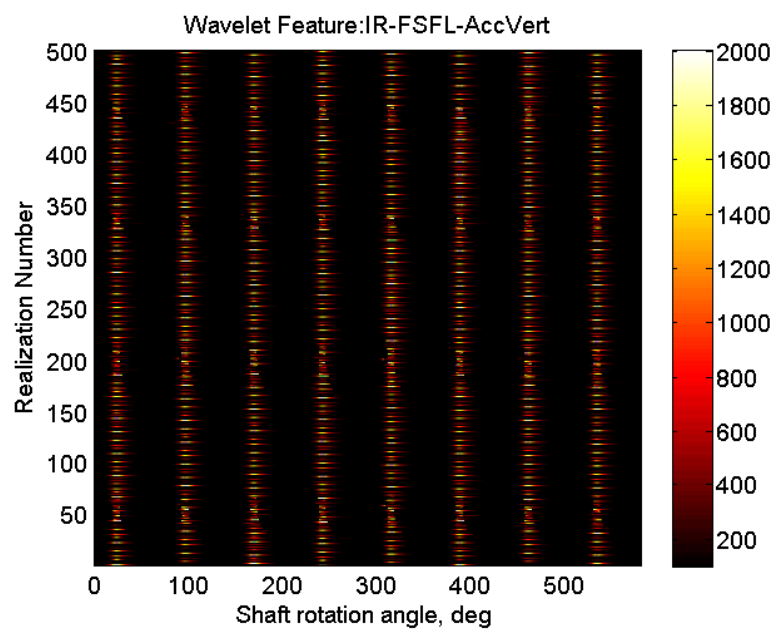

(b)

Figure 8
Figure 10 show diagnostic feature maps of defective bearing and Fisher criterion in frequency domain for full speed and half load test condition. In this case also, the gain in terms of FFT amplitude at defect frequency is 2.85 times with SK based frequency band selection.

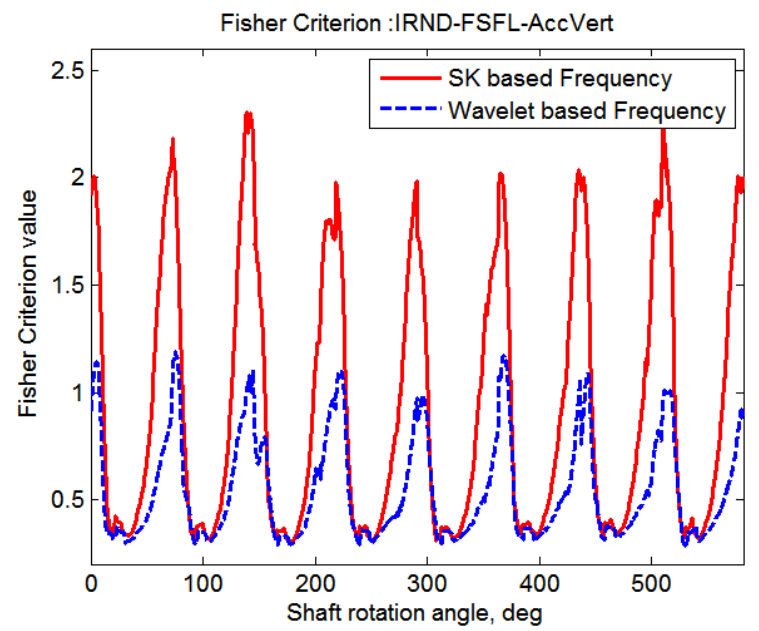

(a)

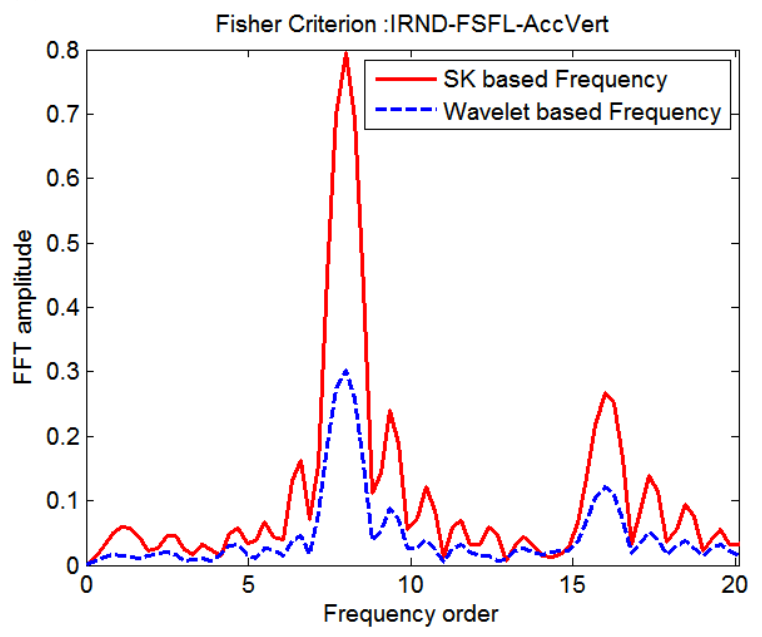

Figure 9 


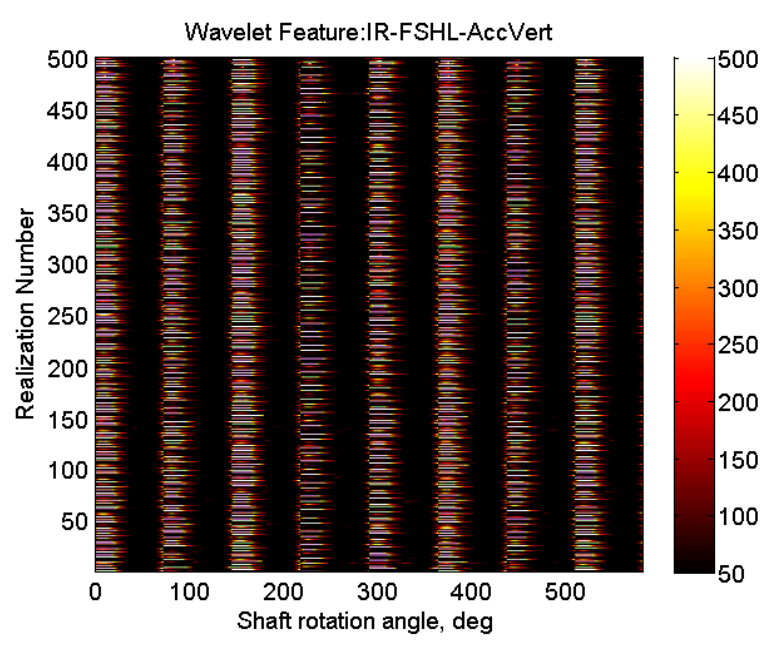

(a)

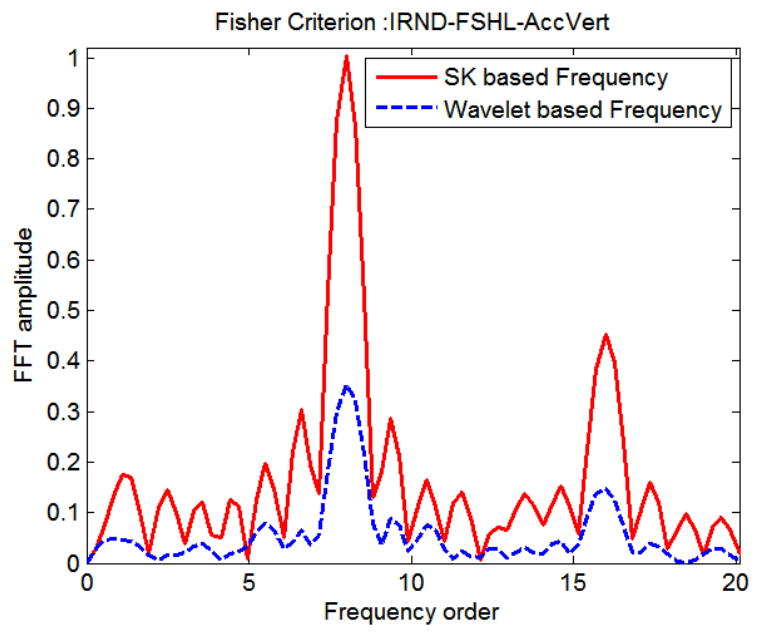

(b)

Figure 10

\section{CONCLUSION}

The novel diagnosis technology combining the spectral kurtosis and wavelet transform is proposed and experimentally validated for bearing defect diagnosis. The spectral kurtosis is used as a pre-processing technique for resonant frequency band selection. The novel signal processing is done periods by periods, enabling it to adapt the stochastic nature of the bearing signal and also remains insensitive to the ball/roller slippage in the bearing raceways.

A diagnostic feature in the form of the normalized instantaneous integrated wavelet modulus is used for bearing condition monitoring. By using comprehensive laboratory experimental data, the experimental validation is performed for early diagnosis of inner race defect $(1.2 \%$ is the relative damage size). The average gain in terms of the Fisher criterion for the proposed technology is 2.75 times compared to the wavelet technology (Gelman et al 2013).
Thus, we recommend usage of the SK as a pre-processing tool for the wavelet diagnosis technology.

The use of SK together with the processing of relatively shorter signal, i.e. only few realizations, makes the proposed technology computationally efficient and could easily be implemented for on-line application. The conventional demodulation technique would need a longer signal in comparison. However, a comparison of the diagnostic potential of the proposed technique with the demodulation technique is required and will be pursued as a future work. The other issue need an attention is the effect of resampling on the performance of the proposed method, particularly in presence of random slippage of rolling element of bearing.

\section{REFERENCES}

Nikolaou, N.G., and Antoniadis, I.A. (2002). Demodulation of vibration signals generated by defects in rolling element bearings using complex shifted Morlet wavelets. Mechanical Systems and Signal Processing, vol. 16, pp. 677-694. doi:10.1006/mssp.2001.1459

Klepka, A. (2011). Wavelet based signal demodulation technique for bearing fault detection. Mechanics and Mechanical Engineering, vol. 15, pp. 63-71.

Qiu, H. , Lee, J., Lin, J., and Yu, G. (2006). Wavelet filter based weak signature detection method and its application on rolling element bearing prognostics. Journal of Sound and Vibration, vol. 289, pp. 10661090. doi:10.1016/j.jsv.2005.03.007

Sawalhi, N., and Randall, R. (2004). The application of spectral kurtosis to bearing diagnostics. Proceedings of ACOUSTICS, November 3-5, Gold Coast, Australia, pp. 393-398.

Antoni, J. (2006). The spectral kurtosis: A useful tool for characterizing non-stationary signals. Mechanical Systems and Signal Processing, vol. 20, pp. 282-520. doi:10.1016/j.ymssp.2004.09.001

Wang, Y., and Liang, M. (2011). An adaptive SK technique and its application for fault detection of rolling element bearings. Mechanical Systems and Signal Processing, vol. 25, pp. 1750-1764 doi:10.1016/j.ymssp.2010.12.008

Gryllias, K.C., Gelman, L., Shaw, B., and Vaidhianathasamy, M. (2010). Local damage diagnosis in gearboxes using novel wavelet technology. Insight, vol. 52(8), pp. 437-441. doi: 10.1784/insi.2010.52.8.437

Gelman, L., Murray, B., Patel, T., and Thomson, A. (2013). The novel wavelet technology for vibration condition monitoring of rolling element bearings, Accepted in Insight. 